\title{
Cognitive variations among vascular dementia subtypes caused by small-, large-, or mixed-vessel disease
}

Huang Ying, Chen Jianping, Yuan Jianqing, Zhong Shanquan

Department of Neurology, the First Affiliated Hospital, Gan Nan Medical College, Jiangxi, China

Submitted: 31 August 2014

Accepted: 20 October 2014

Arch Med Sci 2016; 12, 4: 747-753

DOI: 10.5114 /aoms.2016.60962

Copyright $\odot 2016$ Termedia \& Banach

\section{Abstract}

Introduction: Vascular dementia ( $\mathrm{VaD})$ is a heterogeneous disease that can vary in clinical presentation and cognitive profile. The cognitive profiles of different $\mathrm{VaD}$ subtypes depend on the anatomical distribution of the vascular insults that have been documented.

Material and methods: We reviewed demographic, cognitive, and imaging data in 402 patients who were clinically diagnosed with VaD between January 2002 and June 2012 at the First Affiliated Hospital of Gan Nan Medical College in Ganzhou, China.

Results: Based on magnetic resonance imaging (MRI) results, patients were classified as having large- (24.1\%), small- (70.4\%), or mixed-vessel VaD (5.5\%). Hypertension was the most prevalent risk factor $(81 \%)$, followed by smoking (37\%), hyperlipidemia (35\%), and diabetes (27\%). Hyperlipidemia, cardiac risk factors (history of cardiovascular disease, heart valve disorder) and carotid stenosis were more frequent in patients with large-vessel disease compared to those with small-vessel or mixed-vessel disease $(p<0.001)$. A median of 4 (maximum 11) cognitive domains were impaired in each VaD patient. After memory dysfunction, executive defects were the most prevalent (68.9\%), and neurobehavioral dysfunction was the most rare (13.2\%). Patients with small-vessel VaD showed more executive dysfunction than patients with large-vessel and mixed-vessel $\mathrm{VaD}(p<0.05)$, whereas patients with large-vessel $\mathrm{VaD}$ had a higher prevalence of visuospatial or language dysfunction $(p<0.05)$.

Conclusions: The results indicate that specific subtypes and underlying vascular mechanisms will help predict clinical courses and produce more focused treatment and prevention of VaD.

Key words: vascular dementia, domain, cognition.

\section{Introduction}

Vascular dementia $(\mathrm{VaD})$ is a common type of dementia that is affecting a growing number of elderly individuals worldwide [1, 2]. It is the second leading cause of dementia among the elderly in China; VaD is responsible for more than $25 \%$ of all the dementia cases and is only surpassed in prevalence by Alzheimer's disease (AD) [3, 4].

Vascular dementia is a heterogeneous disease that can vary in clinical presentation and cognitive profile. $\mathrm{VaD}$ can be caused by small-vessel disease (characterized by lacunes, white matter hyperintensities, or bilateral thalamic lesions), large-vessel disease (large territorial or strategic infarcts) $[5,6]$, or mixed-vessel disease. The cognitive profiles of different

\author{
Corresponding author: \\ Huang Ying MD \\ Department of Neurology \\ the First Affiliated Hospital \\ Gan Nan Medical College \\ 23 Qingnian Road \\ Ganzhou, Jiangxi \\ 341000, China \\ Phone: +861015279797688 \\ Fax: +86 108266013 \\ E-mail: huangying0202@126. \\ com
}


VaD subtypes depend on the anatomical distribution of the vascular insults. For example, executive dysfunction and mild memory deficits were proposed as the major part of subcortical ischemic VaD [7]. Previous studies reported that subcortical vascular disease was associated with deficits in working memory, visuomotor speed, and executive dysfunction [8]. Therefore, we hypothesized that $\mathrm{VaD}$ patient cognitive profiles offer clues to the type of underlying vascular disease and thus may be a useful diagnostic tool.

The aims of this study were to characterize cognitive function profiles with respect to domain dysfunction in different subtypes of $\mathrm{VaD}$ patients using neuropsychological tests and assessing magnetic resonance imaging (MRI) using well-defined radiological criteria [6].

\section{Material and methods}

We analyzed the medical records of 402 patients who were diagnosed with $\mathrm{VaD}$ between January 2002 and June 2012 in our hospital. The patients were eligible for inclusion according to the following criteria: age $\geq 40$ years; fulfillment of the NINDSAIREN criteria for probable VaD [9]; MRI evidence of ischemic cerebrovascular disease, including ischemic stroke or white matter lesions; a Mini-Mental State Examination (MMSE) score of 10-26 [10]; and a score of 4 or more on the modified Hachinski Ischemia Scale (HIS) [11]. Patients diagnosed with any primary neurodegenerative disorders, space-occupying lesions, or lobar hemorrhages were excluded, as were patients without complete clinical evaluation and brain imaging. This retrospective study protocol was approved by the Clinical Research Ethics Committee of our hospital.

We collected the following data for each patient: demographic variables, including age, sex, and education; risk factors for dementia, including hypertension, diabetes mellitus, hyperlipidemia, cerebrovascular disease, cardiovascular disease, heart valve disorder, carotid stenosis, or peripheral vascular disease. Patients were also asked if they had a history of smoking. All patients had undergone MRI.

All patients were initially evaluated with the MMSE [10], followed by assessments of neurobehavioral function, memory, executive function, orientation, attention, abstract thinking, calculation, language function, mental control, praxis-gnosis, and visuospatial function. Attention and abstract thinking were respectively evaluated by the Digit Span Forward test and picture completion from Choynowski's Memory Scale [12]. Calculation, orientation, and visuospatial function were assessed by the arithmetic, spatial and temporal, and crossing-pentagons items from the MMSE, respectively [10]. Cortical functional domains were assessed with selected cognitive tests. Language fluency was tested by category naming of animals in $1 \mathrm{~min}$ [13], and praxis-gnosis was estimated with Western Aphasia Battery ideomotor apraxia subtest items [14]. Memory was assessed by the Wechsler Memory Scale (WMS) [15], and mental control and executive functions were evaluated with the revised Wechsler Memory Scale (WMS-R) [16] and trail-making test B (TMT-B), respectively [17]. Neurobehavioral function was evaluated with the caregiver-based 12-item Neuropsychiatric Inventory (NPI) [18]. The specific neuropsychological battery included the HIS [11].

Vascular abnormality assessment included the items of the radiological NINDS-AIREN criteria for VaD according to previously proposed operational definitions [5]. Magnetic resonance imaging (MRI) assessment was performed using well-defined radiological diagnosis by Jellinger in 2008 [6]. Based on these criteria, patients were classified as having large-vessel VaD (strategic large-vessel infarct of the dominant hemisphere or bilateral hemispheric strokes), small-vessel VaD (white-matter hyperintensities involving $\geq 25 \%$ of white matter, multiple lacunes, or bilateral thalamic lesions) or mixed-vessel VaD. Magnetic resonance imaging was performed on a 1.5-Tesla machine (Magnetom Impact Expert Siemens AG, Erlangen, Germany) following a standard protocol, including coronal T1-weighted 3D magnetization-prepared rapid acquisition with gradient echo (MPRAGE), axial fluid-attenuated inversion recovery (FLAIR), and axial spin-echo T2-weighted images.

\section{Statistical analysis}

All statistical analyses were performed using the SPSS computerized statistics package (version 17.0; Chicago, IL, USA). Baseline demographics were analyzed using descriptive statistics. Differences in baseline characteristics and risk factors among the different types of $\mathrm{VaD}$ groups (small-vessel $\mathrm{VaD}$, large-vessel $\mathrm{VaD}$ or mixed small/large-vessel $\mathrm{VaD}$ ) were analyzed using the $\chi^{2}$ test for dichotomous outcome variables and one-way analysis of variance (ANOVA), and Newman-Keuls tests (NK) were applied to continuous data. The $\chi^{2}$ tests were subsequently used to compare impaired cognitive domains among the different $\mathrm{VaD}$ groups. The results of statistical tests were considered significant at the level of $p<0.05$.

\section{Results}

Patient demographics and risk factors are shown in Table I. The total study population included $402 \mathrm{VaD}$ patients (mean age: 69 years), and the mean MMSE score of the patients was 16 . Based on the operational definitions for the ra- 
Table I. Demographics and risk factors of VaD subtypes

\begin{tabular}{|c|c|c|c|c|c|}
\hline Parameter & $\begin{array}{l}\text { Small-vessel } \\
\text { VaD }\end{array}$ & $\begin{array}{l}\text { Large-vessel } \\
\text { VaD }\end{array}$ & $\begin{array}{c}\text { Mixed-vessel } \\
\text { VaD }\end{array}$ & $\begin{array}{l}\chi^{2} \text { or } \\
F \text {-value }\end{array}$ & $\begin{array}{l}\text { Overall unadjusted } \\
p \text {-value }\end{array}$ \\
\hline No. of patients (\%) & $283(70.4)$ & $97(24.1)$ & $22(5.5)$ & & \\
\hline Age, mean \pm SD [years] & $68.3 \pm 7.1$ & $70.5 \pm 9.2^{\mathrm{a}}$ & $69.3 \pm 8.4$ & 3.98 & 0.026 \\
\hline Female, $n(\%)$ & $92(31.8)$ & $35(36.1)$ & $11(50.0)$ & 0.24 & 0.814 \\
\hline Male, $n(\%)$ & $191(68.2)$ & $62(63.9)$ & $11(50.0)$ & & \\
\hline Education, mean \pm SD [years] & $9.6 \pm 3.5$ & $8.2 \pm 2.1$ & $9.3 \pm 2.2$ & 2.23 & 0.133 \\
\hline MMSE, mean \pm SD & $15.7 \pm 3.2$ & $17.4 \pm 3.4$ & $18.6 \pm 4.1$ & 1.65 & 0.476 \\
\hline Hypertension, $n(\%)$ & 226 (79.9) & $81(83.5)$ & $18(81.8)$ & 0.13 & 0.963 \\
\hline Diabetes mellitus, $n$ (\%) & $71(25.1)$ & $29(29.9)$ & $7(31.8)$ & 1.88 & 0.435 \\
\hline Hyperlipidemia, $n$ (\%) & $85(30.0)^{b, c}$ & $48(49.5)$ & $9(40.9)$ & 4.69 & $<0.001$ \\
\hline Cardiovascular disease, $n$ (\%) & $36(12.7)$ & $29(29.9)^{\mathrm{a}, \mathrm{c}}$ & $3(13.6)$ & 5.27 & $<0.001$ \\
\hline Heart valve disorder, $n$ (\%) & $39(13.8)$ & $40(41.2)^{a, c}$ & $3(13.6)$ & 8.33 & $<0.001$ \\
\hline Carotid stenosis, $n(\%)$ & $23(8.1)$ & $21(22.7)^{\mathrm{a}, \mathrm{c}}$ & $2(9.1)$ & 6.48 & $<0.001$ \\
\hline $\begin{array}{l}\text { Peripheral vascular disease, } \\
n(\%)\end{array}$ & $17(6.0)$ & $6(6.2)$ & $2(9.1)$ & 1.01 & 0.662 \\
\hline Smoking, $n(\%)$ & $110(38.9)$ & $37(38.1)$ & $9(40.9)$ & 0.16 & 0.893 \\
\hline
\end{tabular}

MMSE - Mini-Mental State Examination. ${ }^{a}$ Significantly different compared to patients with small-vessel VaD, ${ }^{b}$ significantly different compared to patients with large-vessel VaD, 'significantly different compared to patients with mixed-vessel VaD.

diological component of the NINDS-AIREN criteria, $283(70.4 \%)$ patients had small-vessel VaD, 97 (24.1\%) had large-vessel VaD, and 22 (5.5\%) had mixed-vessel VaD. Patients with large-vessel VaD were older compared with patients with small-vessel $\mathrm{VaD}(p<0.05)$. MMSE scores were comparable among the three groups. Overall, hypertension was the most prevalent risk factor (81\%), followed by smoking (37\%), hyperlipidemia (35\%), and diabetes (27\%). Hyperlipidemia, cardiac risk factors (history of cardiovascular disease, heart valve disorder) and carotid stenosis were more frequent in

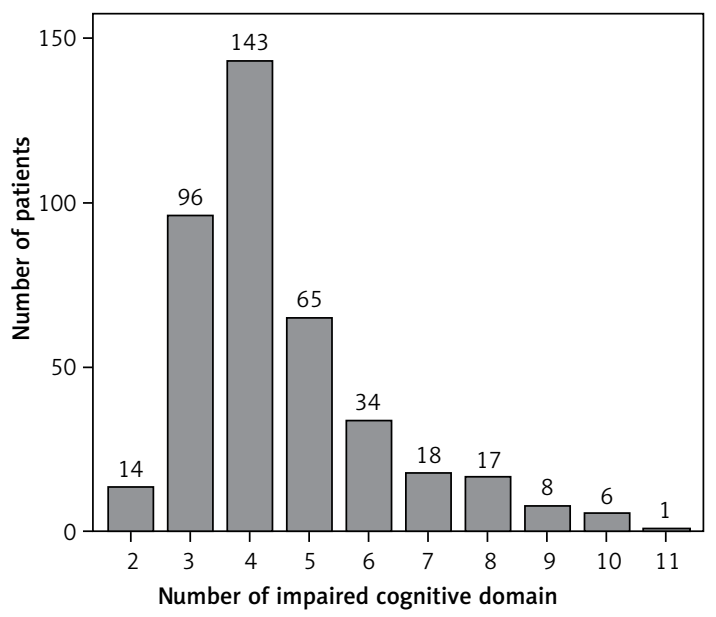

Figure 1. Frequency distribution of the number of impaired cognitive domains per patient $(n=402)$ patients with large-vessel disease compared to those with small-vessel or mixed-vessel disease $(p<0.001)$; but smoking, peripheral vascular disorder and diabetes were equally distributed among the different $\mathrm{VaD}$ subtypes.

The median was 4 impaired cognitive domains per patient (Figure 1). However, the number of impaired cognitive domains did not differ among $\mathrm{VaD}$ patient groups $(p=0.36)$. In all, we examined 11 cognitive domains (Figure 2). Besides memory defect, executive dysfunction was the most common (68.9\%), followed by calculation defects

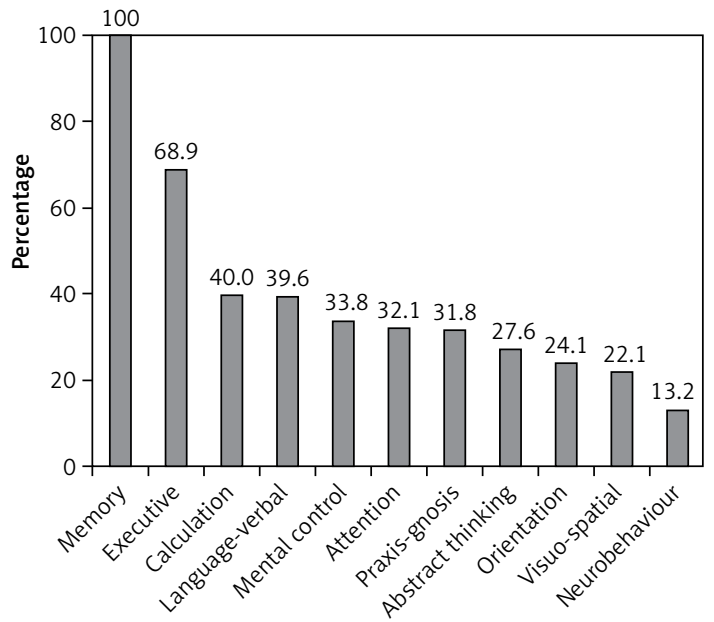

Figure 2. Percentages of patients presenting individual cognitive domain impairment $(n=402)$ 
(40.5\%), language dysfunction (39.6\%), and mental control dysfunction (33.8\%). Neurobehavioral defects were the least common (13.2\%). The relative prevalence of executive, visuospatial, and language dysfunction was significantly different among the different $\mathrm{VaD}$ subtypes $(p<0.001)$ (Table II). By using logistic regression models controlling for age, we found that executive dysfunction was more prevalent in patients with small-vessel VaD compared with patients with large-vessel and mixed-vessel $\operatorname{VaD}(p<0.05)$. Notably, patients with large-vessel $\mathrm{VaD}$ more frequently exhibited visuospatial and language-verbal dysfunction $(p<0.05)$. Other cognitive domain impairments, including memory, orientation, calculation, abstract thinking, praxis-gnosis, mental control, and attention dysfunction were equally distributed among the three $\mathrm{VaD}$ patient subtypes.

\section{Discussion}

The main findings of the present study are that the domain profile of cognitive dysfunction differs among $\mathrm{VaD}$ subtypes, and that these subtypes are associated with specific risk factors. Specifically, we demonstrated that executive dysfunction is more prevalent in patients with small-vessel VaD compared with large- and mixed-vessel $\mathrm{VaD}$, whereas language and visuospatial dysfunction were more often observed in patients with large-vessel VaD.

As the biological dementia process begins years before it is diagnosed clinically, the preclinical period of the disease, when neuronal degeneration has begun, but cellular and biochemical damage is not yet sufficient for symptoms to manifest, is the optimal time to initiate a neuroprotective therapy [19]. Currently molecular imaging methods are state-of-the-art to diagnose dementia [20]. For example, MRI enabled the visualization of specific structures within the medial temporal lobe, such as the hippocampus itself, the parahippocampal gyrus, subiculum, entorhinal cortex, and amygdale, with good clinical correlations with dementia [21]. Resting-state functional magnetic resonance imaging detected that amplitude low-frequency fluctuations of the right angular gyrus in the slow5 band are more specific for diagnosing subcortical ischemic vascular disease [22]. Hence, the differences in patterns of cognitive domain deficits between the subtypes in $\mathrm{VaD}$ could be explained by the heterogeneity with regard to the location of lesions. Medial temporal location is consistent with the characteristic amnestic state in large-vessel VaD [23], whereas basal ganglia infarcts usually produce executive dysfunction [24]. Alternatively, infarcts in the thalamus, which is an important area in the episodic memory circuit, could cause memory impairment in some subcortical VaD patients [23]. Executive dysfunction in VaD patients with extensive white matter hyperintensities may be explained by frontal hypometabolism, which was mostly characterized by weakened goal-directed actions [25]. Poor working memory and executive dysfunction in subcortical VaD may be the result of disrupted frontal-subcortical circuits [26].

The difference between $\mathrm{VaD}$ subtype cognitive profiles may be explained, on one hand, by the anatomical distribution of the vascular insults. For example, Alladi et al. [27] reported that small artery disease was more often associated with subcortical VaD than with cortical and cortical-subcortical VaD. It has been suggested that subcortical $\mathrm{VaD}$ is homogeneous, with small-vessel disease as the primary vascular etiology, lacunar infarcts

Table II. Prevalence of cognitive domain impairment by VaD subtype

\begin{tabular}{|lcccc|}
\hline Variable & $\begin{array}{c}\text { Small-vessel } \\
\text { VaD } \\
(n=283)\end{array}$ & $\begin{array}{c}\text { Large-vessel } \\
\text { VaD } \\
(n=97)\end{array}$ & $\begin{array}{c}\text { Mixed-vessel } \\
\text { VaD } \\
(n=22)\end{array}$ & $\begin{array}{c}\text { Chi-square overall } \\
\text { unadjusted } \\
P \text {-value }\end{array}$ \\
\hline Abstract thinking & $77(27.2 \%)$ & $30(30.9 \%)$ & $4(18.2 \%)$ & 0.464 \\
\hline Attention & $88(31.1 \%)$ & $35(36.1 \%)$ & $6(27.3 \%)$ & 0.480 \\
\hline Calculation & $116(41.0 \%)$ & $43(44.3 \%)$ & $4(18.2 \%)$ & 0.334 \\
\hline Executive & $223(78.8 \%)^{b, c}$ & $38(39.2 \%)$ & $16(72.7 \%)$ & $<0.001$ \\
\hline Language & $94(33.2 \%)$ & $57(58.8 \%)^{a}$ & $8(36.4 \%)$ & $<0.001$ \\
\hline Mental control & $96(33.9 \%)$ & $33(34.0 \%)$ & $7(31.8 \%)$ & 0.979 \\
\hline Neurobehavior & $35(12.4 \%)$ & $16(16.5 \%)$ & $2(9.1 \%)$ & 0.493 \\
\hline Orientation & $70(24.7 \%)$ & $19(19.6 \%)$ & $8(36.4 \%)$ & 0.229 \\
\hline Praxis-gnosis & $95(33.6 \%)$ & $26(26.8 \%)$ & $7(31.8 \%)$ & 0.467 \\
\hline Visuospatial & $44(15.5 \%)$ & $36(37.1 \%)^{a, c}$ & $9(40.9 \%)$ & $<0.001$ \\
\hline
\end{tabular}

aSignificantly different compared to patients with small-vessel VaD, ${ }^{b}$ significantly different compared to patients with large-vessel VaD, csignificantly different compared to patients with mixed-vessel VaD. 
Table III. Performance of magnetic resonance imaging in subtypes of vascular dementia (VaD)

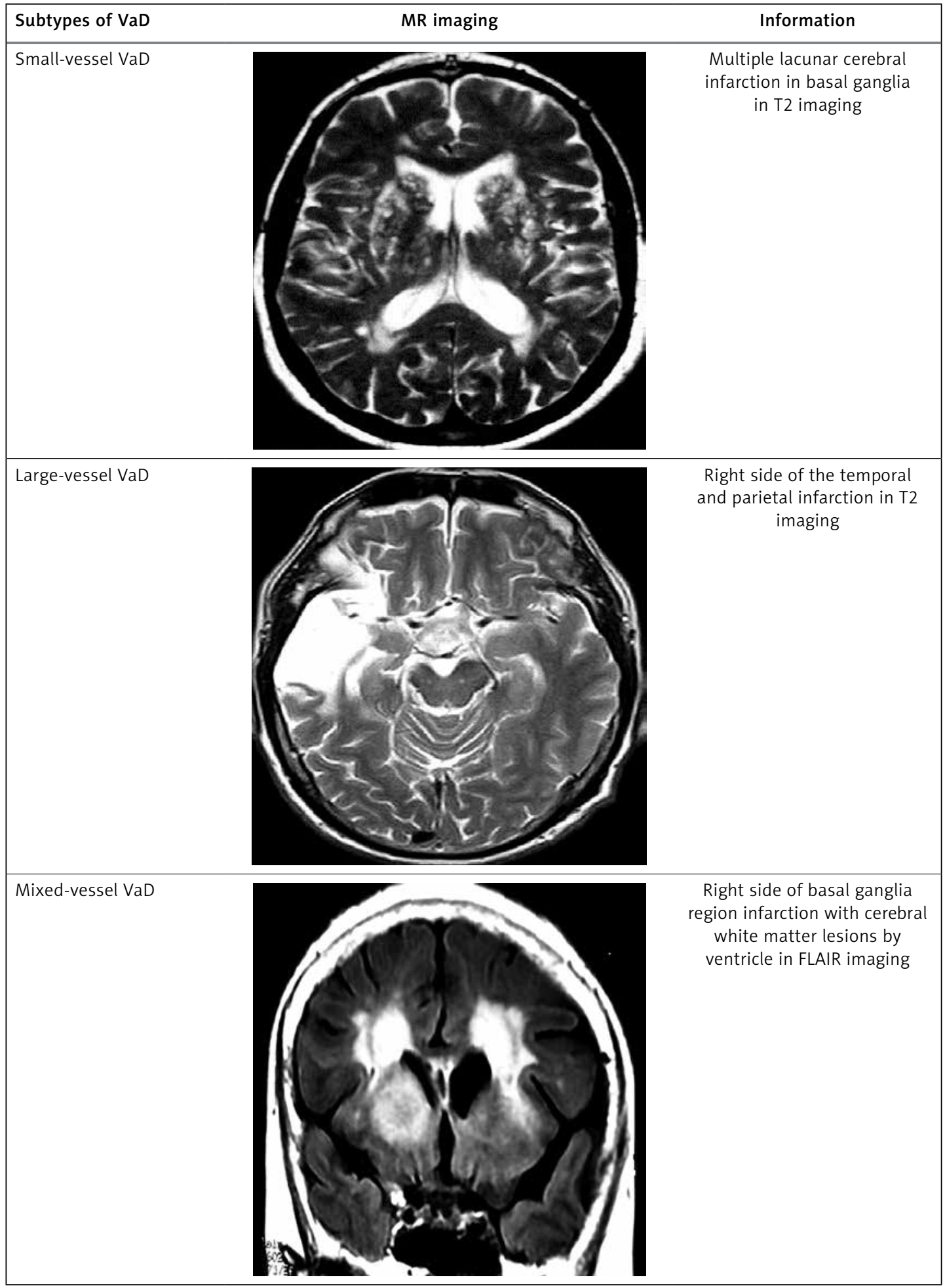

and ischemic white matter lesions as the primary type of brain lesions, and subcortical clinical syndrome as the primary clinical manifestation. Consistent with our results, previous studies reported that subcortical VaD was associated with working memory deficits, visuomotor speed and executive dysfunction [28]. The VaD patients with extensive white matter hyperintensities or multiple lacunar infarctions also showed executive dysfunction [29]. On the other hand, neurotransmitters have been reported to be decreased in dementia patients, especially in the case of acetylcholine, which is a potent vasodilator innervating local cortical nitroxidergic interneurons, cerebral 
capillaries and microarterioles. A clinical study also found that acetylcholine esterase inhibitor increased the basal cerebral blood flow and could be used to treat dementia [30]. So, the nonuniform cholinergic cortical innervation could explain the regional profile variability in dementia patients.

At the same time, our study examined the relative proportion of small-vessel disease of $\mathrm{VaD}$ in the southern Chinese Jiangxi Province, which has a population of 900 million people, an area of 39,400 square kilometers and a subtropical humid monsoon climate. Similar to our results, a cross-sectional community-based study of 706 $\mathrm{VaD}$ patients reported relative prevalences of $74 \%$, $18 \%$, and $8 \%$ for small-, large-, and mixed-vessel disease, respectively [31]. A previous neuropathological study provided support for the view that small-vessel disease is the main component of $\operatorname{VaD}[32]$.

Given that this is a retrospective study, there is a lack of data to evaluate cognitive dysfunction severity, and there is also a selection bias. In addition, due to the lack of integrated data, the effects of physical disabilities on cognition among vascular dementia subtypes were not determined. Therefore, further prospective clinical research is needed, particularly in order to evaluate the severity of cognitive dysfunction and the effects of physical disabilities on cognition by MRI and neuropsychological examinations in VaD. Future studies are also required to predict the course of disease by specifying the subtypes and underlying vascular mechanisms using MRI and develop cutting edge tools and concepts for constructing a VaD model, like that of Sun et al. [33] (Table III).

\section{Conflict of interest}

The authors declare no conflict of interest.

\section{References}

1. Roman GC. Stroke, cognitive decline and vascular dementia: the silent epidemic of the 21st century. Neuroepidemiology 2003; 22: 161-4.

2. Kalaria RN, Maestre GE, Arizaga R, et al. Alzheimer's disease and vascular dementia in developing countries: prevalence, management, and risk factors. Lancet Neurol 2008; 7: 812-26.

3. Zhang ZX, Zahner GE, Roman GC, et al. Dementia subtypes in China: prevalence in Beijing, Xian, Shanghai, and Chengdu. Arch Neurol 2005; 62: 447-53.

4. Dong MJ, Peng B, Lin XT, Zhao J, Zhou YR, Wang RH. The prevalence of dementia in the People's Republic of China: a systematic analysis of 1980-2004 studies. Age Ageing 2007; 36: 619-24.

5. van SEC, Scheltens P, Knol DL, et al. Operational definitions for the NINDS-AIREN criteria for vascular dementia: an interobserver study. Stroke 2003; 34: 1907-12.

6. Jellinger KA. Morphologic diagnosis of "vascular dementia" - a critical update. J Neurol Sci 2008; 270: 1-12.
7. Erkinjuntti T, Inzitari D, Pantoni L, et al. Research criteria for subcortical vascular dementia in clinical trials. J Neural Transm Suppl 2000; 59: 23-30.

8. Traykov L, Baudic S, Thibaudet MC, Rigaud AS, Smagghe A, Boller F. Neuropsychological deficit in early subcortical vascular dementia: comparison to Alzheimer's disease. Dement Geriatr Cogn Disord 2002; 14: 26-32.

9. Roman GC, Tatemichi TK, Erkinjuntti T, et al. Vascular dementia: diagnostic criteria for research studies. Report of the NINDS-AIREN International Workshop. Neurology 1993; 43: 250-60.

10. Folstein MF, Folstein SE, McHugh PR. "Mini-mental state". A practical method for grading the cognitive state of patients for the clinician. J Psychiatr Res 1975; 12: $189-98$

11. Rosen WG, Terry RD, Fuld PA, Katzman R, Peck A. Pathological verification of ischemic score in differentiation of dementias. Ann Neurol 1980; 7: 486-8.

12. Janowski K, Gustaw K, Kasprowicz M. Application of Choynowski's Memory Scale in assessment of patients with dementia. Arch Med Sci 2012; 8: 130-7.

13. Welsh KA, Butters N, Mohs RC, et al. The Consortium to Establish a Registry for Alzheimer's Disease (CERAD). Part V. A normative study of the neuropsychological battery. Neurology 1994; 44: 609-14.

14. Kertesz A. Western Aphasia Battery. The Psychological Corporation, San Antonio 1983.

15. Wechsler DA. Standardized memory scale for clinical use. J Psychol 1945; 19: 87-95.

16. Wechsler D. Wechsler Memory Scale-Revised. The Psychological Corporation, San Antonio 1987.

17. Reitan RM. Validity of the trail making test as an indicator of organic brain damage. Percept Mot Skills 1958; 8: 271-6.

18. Cummings JL, Mega M, Gray K, Rosenberg-Thompson S, Carusi DA, Gornbein J. The Neuropsychiatric Inventory: comprehensive assessment of psychopathology in dementia. Neurology 1994; 44: 2308-14.

19. Mosconi L. Brain glucose metabolism in the early and specific diagnosis of Alzheimer's disease FDG-PET studies in MCl and AD. Eur J Nucl Med Mol Imaging 2005; 32: 486-516.

20. Schaller BJ. Strategies for molecular imaging dementia and neurodegenerative diseases. Neuropsychiatr Dis Treat 2008; 4: 585-612.

21. Chong MS, Sahadevan S. Preclinical Alzheimer's disease: diagnosis and prediction of progression. Lancet Neurol 2005; 4: 576-9.

22. Li C, Liu C, Yin X, et al. Frequency-dependent changes in the amplitude of low-frequency fluctuations in subcortical ischemic vascular disease (SIVD): a resting-state fMRI study. Behav Brain Res 2014; 4: 205-10.

23. Aggleton JP, Brown MW. Episodic memory, amnesia, and the hippocampal-anterior thalamic axis. Behav Brain Sci 1999; 22: 425-44; discussion 444-89.

24. Leys D, Henon H, Mackowiak-Cordoliani MA, Pasquier F. Poststroke dementia. Lancet Neurol 2005; 4: 752-9.

25. Tullberg M, Fletcher E, DeCarli C, et al. White matter lesions impair frontal lobe function regardless of their location. Neurology 2004; 63: 246-53.

26. Reed BR, Eberling JL, Mungas D, Weiner MW, Jagust WJ. Memory failure has different mechanisms in subcortical stroke and Alzheimer's disease. Ann Disord 2003; 17: 236-42.

27. Alladi S, Kaul S, Meena AK, Somayajula S, Umadevi M, Reddy JM. Pattern of vascular dementia in India: study of clinical features, imaging, and vascular mechanisms 
from a hospital dementia registry. J Stroke Cerebrovasc Dis 2006; 15: 49-56.

28. Pohjasvaara T, Mantyla R, Ylikoski R, Kaste M, Erkinjuntti T. Clinical features of MRI-defined subcortical vascular disease. Alzheimer Dis Assoc Disord 2003; 17: 236-42.

29. Cummings JL, Mega M, Gray K, Rosenberg-Thompson S, Carusi DA, Gornbein J. The Neuropsychiatric Inventory: comprehensive assessment of psychopathology in dementia. Neurology 1994; 44: 2308-14.

30. Schaller B. Prospects for the future: the role of free radicals in the treatment of stroke. Free Radic Biol Med 2005; 38: 411-25.

31. Staekenborg SS, van SEC, der Flier WM, Lane R, Barkhof F, Scheltens P. Small vessel versus large vessel vascular dementia: risk factors and MRI findings. J Neurol 2008; 255: 1644-51; discussion 1813-4.

32. Esiri MM, Wilcock GK, Morris JH. Neuropathological assessment of the lesions of significance in vascular dementia. J Neurol Neurosurg Psychiatry 1997; 63: 749-53.

33. Sun C, Zhang H, Xu J, et al. Improved methodology to obtain large quantities of correctly folded recombinant $\mathrm{N}$-terminal extracellular domain of the human muscle acetylcholine receptor for inducing experimental autoimmune myasthenia gravis in rats. Arch Med Sci 2014 10: 389-95. 